\title{
Pelvic Floor Muscle Weakness, CTCAE
}

National Cancer Institute

\section{Source}

National Cancer Institute. Pelvic Floor Muscle Weakness, CT CAE. NCI Thesaurus. Code C143739.

A disorder characterized by a reduction in the strength of the muscles of the pelvic floor. 